\title{
Comportamento da função do ventrículo esquerdo a longo prazo no transplante cardíaco ortotópico
}

Alfredo Inácio FIORELLI*, Noedir A. G. STOLF*, Pedro Carlos Piantino LEMOS*, Pablo M. A. POMERANTZEFF*, Edmar A. BOCCHI*, Pedro GRAZIOZI*, Fábio BUSNARDO*, Fábio GAIOTTO*, Marisa Fernandes Silva GÓES*, Adib D. JATENE*

RBCCV 44205-204

FIORELLI, A. I.; STOLF, N. A. G.; LEMOS, P. C. P.; POMERANTZEFF, P. M. A.; BOCCHI, E. A.; GRAZIOZI, P.; BUSNARDO, F.; GAIOTO, F.; GÓES, M. F. S.; JATENE, A. D. - Comportamento da função do ventrículo esquerdo a longo prazo no transplante cardíaco ortotópico. Rev. Bras. Cir. Cardiovasc., $8(2): 97-107,1993$.

RESUMO: No InCór-FMUSP, no período de março de 1985 a fevereiro de 1993 (75 meses), 110 pacientes portadores de miocardiopatia terminal foram submetidos a transplante cardiaco ortotópico (TCO). Desta série, foram selecionados 24 pacientes, com sobrevida superior a 36 meses, para análise da função do ventrículo esquerdo (VE) a longo prazo. O período de seguimento clínico esteve compreendido entre 36 meses e 78 meses (56 meses). A idade variou de 27 a 60 anos ( 44,3 anos), sendo 21 ( $87,5 \%$ ) do sexo masculino. As etiologias da doença cardiaca que motivaram a indicação dos transplantes foram: miocardiopatia dilatada em $13(54,2 \%)$ pacientes, isquêmica em $8(33,3 \%)$ e chagásica em $3(12,5 \%)$. Todos encontravamse, inicialmente, em classe funcional IV (NYHA). A cinecoronariografia e as variáveis hemodinâmicas e ecocardiográficas foram aferidas, no pós-operatório, anualmente até o quinto ano de evolução. Os resultados obtidos permitem concluir que: 1) o VE, em todos os casos, apresenta hipertrofia como mecanismo de adaptaçāo; 2) a hipertensão arterial esteve presente desde o primeiro ano de evolução; 3) as rejeiçōes agudas e a etiologia da miocardiopatia não interferiram na função do VE; 4) a aterosclerose acelerada exerce efeito negativo na função do VE; 5 ) do ponto de vista ecocardiográfico e hemodinâmico, a função do VE manteve-se estável até o quinto ano de evolução pós-operatória; o aparecimento de redução significativa do índice cardíaco (IC) associado à incidência crescente de resultados anormais nas variáveis analisadas, sugere tendência à depressāo tardia do seu desempenho.

DESCRITORES: transplante cardíaco, ortotópico; transplante cardiaco, função de ventrículo esquerdo.

\section{INTRODUÇÃO}

A despeito do elevado número de transplantes cardíacos até agora realizados, as pesquisas sobre o desempenho do coração transplantado continuam atraindo os investigadores $6,11,15$ desde os estudos pioneiros de CARREL e GUTHRIE ${ }^{4}$, em 1905.

A ciclosporina-A introduziu mudanças nos protocolos de seguimento pós-operatório, considerando-se a maior estabilidade hemodinâmica dos pa- cientes durante os episódios de rejeição e os seus efeitos colaterais $2,7,11$.

Os resultados dos estudos referentes à função do coração e ao comportamento do sistema circulatório são motivo de controvérsias ${ }^{16}$. Os trabalhos, em sua maioria, relatam que a função contrátil do coração desnervado mantém-se inalterada a longo prazo 2, 10, 12; contudo, outros demonstram haver redução importante da função ventricular ${ }^{14}$.

- Do Instituto do Coração do Hospital das Clínicas da Faculdade de Medicina da Universidade de São Paulo.

Endereço para separatas: Alfredo Fiorelli: Av. Dr. Enéas de Carvalho Aguiar, 44. Divisão Cirúrgica. 05403 São Paulo, SP, Brasil. 
FIORELLI, A. I.; STOLF, N. A. G.; LEMOS, P. C. P.; POMERANTZEFF, P. M. A.; BOCCHI, E. A.; GRAZIOZI, P.; BUSNARDO, F.; GAIOTO, F.; GÓES, M. F. S.; JATENE, A. D. - Comportamento da função do ventrículo esquerdo a longo prazo no transplante cardiaco ortotópico. Rev. Bras. Cir. Cardiovasc., 8 (2):97-107, 1993.

O coração transplantado, ao longo de sua evolução, está sujeito à ação de diferentes fatores que podem interferir, tanto no seu desempenho, como na sobrevida do paciente. O tipo de miocardiopatia e o sexo do doador e do receptor parecem não influenciar a função do VE após o transplante. Por outro lado, a hipertensão arterial sistêmica, a fibrose miocárdica, os surtos agudos de rejeiçāo e a aterosclerose coronária têm sido apontados como importantes fatores que interferem a longo prazo na função do coração transplantado; contudo os mecanismos fisiopatológicos responsáveis por tais eventos não estão totalmente elucidados $6,10,11,19,21$.

Poucos estudos sobre a função do coração transplantado têm sido reportados com evolução a longo prazo em pacientes que utilizam a ciclosporinaA na imunossupressão 10,28 . Esses estudos apresentam algumas limitaçōes, posto que é inevitável a redução do número de indivíduos em seguimento com o passar do tempo, principalmente por óbito. Por outro lado, torna-se difícil a exclusão de pacientes com curto período de evolução clínica, pois compromete a casuística a ser analisada.

Considerando-se essas dificuldades, o presente estudo tem por finalidade analisar a evolução anual da função tardia do ventrículo esquerdo (VE) do coração transplantado em situação ortotópica dos pacientes com sobrevida superior a três anos, através de diferentes parâmetros hemodinâmicos e ecocardiográficos.

\section{CASUÍSTICA E MÉTODOS}

No Instituto do Coração do Hospital das Clínicas da Faculdade de Medicina da Universidade de São Paulo (InCór), no período de março de 1985 a dezembro de 1991 (91 meses), 90 pacientes portadores de insuficiência cardiaca em fase terminal foram submetidos ao transplante cardíaco ortotópico. Para o desenvolvimento da presente investigação foram selecionados 24 pacientes desta série, com evolução clínica superior a 36 meses.

A idade dos pacientes variou entre 27 a 60 anos, com média de 44,3 anos e desvio padrão de 7,9 anos. Vinte e um $(87,5 \%)$ pacientes são do sexo masculino e $3(12,5 \%)$ do sexo feminino, sendo 20 $(83,2 \%)$ pacientes da raça branca, $2(8,4 \%)$ da raça negra e $2(8,4 \%)$ pacientes da raça amarela. As etiologias da doença cardíaca que motivaram a indicação dos transplantes foram: miocardiopatia dilatada em $13(54,2 \%)$ pacientes, isquêmica em 8 $(33,3 \%)$ e chagásica em $3(12,5 \%)$ pacientes.

Todos eles encontravam-se, inicialmente, em insuficiência cardíaca grave, em classe funcional IV (NYHA), sendo que $5(20,8 \%)$ pacientes permane- ceram internados, no período pré-operatório, em unidade de terapia intensiva, em uso de catecolaminas por via endovenosa.

O período de evolução clínica pós-operatória esteve compreendido entre 36 e 78 meses, com média de 56 meses, e desvio padrāo de 13,2 meses.

Os corações foram provenientes de doadores cuja idade variou entre 15 e 41 anos, com média de 23,8 anos, e desvio padrão de 6,8 anos. O transplante do órgão foi utilizado em $2(8,3 \%)$ oportunidades, sendo: em $1(4,2 \%)$ caso à longa distância e em outro $(4,2 \%)$ o coração foi retirado em hospital da mesma comunidade. Nos demais $(91,6 \%)$ a cardiectomia foi realizada em sala no mesmo centro cirúrgico do receptor. O tempo de anóxia do órgão variou entre 66 minutos a 165 minutos, com média de 87,2 minutos, e desvio padräo de 22,5 minutos.

O critério de normalidade do coração do doador foi baseado no exame ecocardiográfico em 13 $(54,2 \%)$ casos, no cateterismo cardíaco em 17 $(70,8 \%)$ e em $1(4,2 \%)$ doador, com transporte à longa distância, na inspeção macroscópica do órgão a céu aberto.

Para se avaliar a função do VE foram selecionados todos os pacientes sobreviventes ao transplante cardíaco que se encontram em seguimento pós-operatório superior a $\mathbf{3 6}$ meses, limitando-se a investigação no $60^{\circ}$ mês.

Os principais critérios utilizados na seleção dos receptores e doadores foram os classicamente conhecidos 7, 11. A normalidade do coração a ser transplantado teve por base o exame clínico cardiológico associado ao eletrocardiograma, à radiografia do tórax, ao ecocardiograma e, em situações especiais, ao cateterismo cardíaco.

A imunossupressão imediata, adotada nos 9 $(37,5 \%)$ primeiros pacientes consistiu de ciclosporina-A e predinisona. Um $(4,17 \%)$ paciente recebeu ciclosporina-A e azatioprina, devido ao diabetes melito. Nos demais pacientes $(58,3 \%)$ adotou-se o esquema tríplice com ciclosporina-A, azatioprina $e$ corticóides. Na fase tardia todos os pacientes passaram a receber o esquema tríplice de imunossupressão, cujas doses foram moduladas de acordo com a avaliação clínica.

O estudo da função tardia do VE foi baseado na avaliação ecocardiográfica, hemodinâmica, e na ventriculografia. A cinecoronariografia foi incluida neste estudo para identificar possíveis alterações anatômicas das artérias coronárias. Os exames selecionados foram agrupados nos respectivos anos de evolução, assim denominados A1, A2, A3, A4 e A5.

Foram selecionados 96 estudos hemodinâmicos, 
FIORELLI, A. I.; STOLF, N. A. G.; LEMOS, P. C. P.; POMERANTZEFF, P. M. A.; BOCCHI, E. A.; GRAZIOZI, P.; BUSNARDO, F.; GAIOTO, F.; GÓES, M. F. S.; JATENE, A. D. - Comportamento da função do ventrículo esquerdo a longo prazo no transplante cardíaco ortotópico. Rev. Bras. Cir. Cardiovasc., 8 (2):97-107, 1993.

assim distribuídos: 24 exames com $12,0 \pm 3,5$ meses (A1); $23 \mathrm{com} 24,0 \pm 5,6$ meses (A2); $22 \mathrm{com}$ $36,0 \pm 5,8$ (meses) (A3); 15 com $48,0 \pm 6,1$ meses (A4) e 12 exames com 60,0 $\pm 8,1$ meses (A5). Dois $(8,3 \%)$ pacientes não foram submetidos ao cateterismo cardíaco: um não aceitou o procedimento (caso 16) e o outro (caso 14) apresentava alergia ao iodo.

O estudo hemodinâmico foi realizado no final das biopsias endomiocárdicas, através da introdução do cateter de Swan-Ganz (93A-131-7F) pela mesma via de acesso, ou durante o cateterismo cardíaco anual, incluindo, nestes casos, a pressão diastólica final de VE (pd2VE) e a pressão na aorta (sistólica-PSA, diastólica-PDA e média-PMA). Na ausência deste último exame, a pressão de capilar pulmonar (PCP) foi admitida como representativa da pd2VE, assim como a pressão arterial periférica foi admitida como semelhante à pressão da aorta. Foram selecionados os seguintes parâmetros: a pressão sistólica de artéria pulmonar (PSAP), a PCP, a pd2VE, a PSA, a PDA, a PMA, a resistência vascular sistêmica (RVS) e pulmonar (RVP), e os índices do trabalho sistólico de VE (ITSVE), cardíaco (IC) e sistólico (IS).

$\mathrm{Na}$ avaliação ecocardiográfica foram selecionados 100 exames, assim distribuídos: 24 exames com $12,0 \pm 2,2$ meses (A1); 24 com 24,0 $\pm 2,9$ meses (A2); 24 com 36,0 $\pm 2,1$ meses (A3); $16 \mathrm{com}$ $48,0 \pm 1,3$ meses (A4) e 12 exames com $60,0 \pm 2,1$ meses (A5). As imagens ecocardiográficas obtidas foram analisadas segundo as orientações da American Society of Echocardiography ${ }^{23}$, selecionando-se as seguintes variáveis:

- volume sistólico de VE (VS), valor normal de $38,4 \mathrm{ml}$ a $107,1 \mathrm{ml}$;

- fração de ejeção de VE (FEeco), valor normal acima de 0,65 ;

- índice de massa de VE (IMVE), hipertrofia de VE acima de $100 \mathrm{~g} / \mathrm{m}^{2}$;

- relação massa volume de VE (RVMVE), hipertrofia de VE abaixo de $0,55 \mathrm{ml} / \mathrm{g}$.
Foram, analisados, também, do ponto de vista qualitativo, o aspecto anatomofuncional das valvas cardíacas, considerando-se a mobilidade, o aparecimento de calcificações e a presença de estenose ou insuficiência.

$\mathrm{Na}$ análise evolutiva da função de VE foram excluídos os valores do pré-operatório do paciente, tendo em vista as dificuldades de comparação dos resultados pela mudança do coração. Do mesmo modo, os resultados dos exames do coração do doador também não foram comparados, posto que as suas interpretações são questionáveis, considerando-se a instabilidade hemodinâmica do doador em decorrência da própria morte encefálica.

Para o cálculo da fração de ejeção global do VE (FEcat) foi empregada a ventriculografia esquerda em plano único, na posição oblíqua anterior esquerda a $30^{\circ}$. Valores da FEcat superiores a 0,50 foram admitidos como normais.

No estudo estatístico das variáveis aferidas foi utilizada a análise de variância (Teste F). Para a variável que mostrou resultado significante neste estudo (IC), foi aplicada, a seguir, a análise de variância de dados repetidos, utilizando-se apenas os pacientes que apresentavam dados referentes aos cinco anos consecutivos. Todos os testes tiveram seu valor de significância inferior a 0,05. Todos os cálculos foram realizados empregando-se o programa de análise estatística por computador SAS ("Statistical Analysis System") 24.

\section{RESULTADOS}

Após o transplante, $23(95,8 \%)$ pacientes evoluíram em classe funcional I e $1(4,17 \%)$ em classe funcional II. Neste último paciente, os exames ecocardiográficos realizados a partir do segundo mês de evolução mostravam aumento das câmeras cardíacas com depressão da FEeco. A cinecoronariografia realizada no final do primeiro ano de

TABELA 1

INCIDÊNCIA DE DISFUNÇÃO VALVAR PELA ECOCARDIOGRAFIA (*)

\begin{tabular}{|c|c|c|c|c|c|c|c|}
\hline VALVA & A1 & $A 2$ & $A 3$ & & A4 & & A5 \\
\hline Tricúspide & $\begin{array}{c}2 / 24 \\
(8,3 \%)\end{array}$ & $\begin{array}{c}4 / 24 \\
(16,6 \%)\end{array}$ & $\begin{array}{c}5 / 24 \\
(20,8 \%)\end{array}$ & & $\begin{array}{c}4 / 16 \\
(25,0 \%)\end{array}$ & & $\begin{array}{c}3 / 12 \\
(25,0 \%)\end{array}$ \\
\hline Mitral & $\begin{array}{c}0 / 24 \\
(0,0 \%)\end{array}$ & $\begin{array}{c}0 / 24 \\
(0,0 \%)\end{array}$ & $\begin{array}{c}3 / 24 \\
(12,5 \%)\end{array}$ & & $\begin{array}{c}6 / 16 \\
(37,5 \%)\end{array}$ & 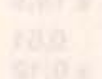 & $\begin{array}{c}2 / 12 \\
(16,6 \%)\end{array}$ \\
\hline Aórtica & $\begin{array}{c}0 / 24 \\
(0,0 \%)\end{array}$ & $\begin{array}{c}1 / 24 \\
(4,2 \%)\end{array}$ & $\begin{array}{c}1 / 24 \\
(4,2 \%)\end{array}$ & & $\begin{array}{c}2 / 16 \\
(12,5 \%)\end{array}$ & $x^{2}=x^{2}$ & $\begin{array}{c}2 / 12 \\
(16,6 \%)\end{array}$ \\
\hline Pulmonar & $\begin{array}{l}0 / 24 \\
(0,0)\end{array}$ & $\begin{array}{c}0 / 24 \\
(0,0 \%)\end{array}$ & $\begin{array}{c}0 / 24 \\
(0,0 \%)\end{array}$ & & $\begin{array}{c}0 / 16 \\
(0,0 \%)\end{array}$ & & $\begin{array}{c}0 / 12 \\
(0,0 \%)\end{array}$ \\
\hline
\end{tabular}

(*) - Insuficiência valvar discreta a moderada. 
FIORELLI, A. I.; STOLF, N. A. G.; LEMOS, P. C. P.; POMERANTZEFF, P. M. A.; BOCCHI, E. A.; GRAZIOZI, P.; BUSNARDO, F.; GAIOTO, F.; GÓES, M. F. S.; JATENE, A. D. - Comportamento da funçāo do ventrículo esquerdo a longo prazo no transplante cardiaco ortotópico. Rev. Bras. Cir. Cardiovasc., 8 (2):97-107, 1993.

seguimento, mostrava intenso comprometimento aterosclerótico nas diversas artérias e a ventriculografia esquerda, hipocinesia difusa de VE.

\section{1 - Avaliação da Ecocardiografia}

A distribuição da incidência da disfunção valvar durante o período de estudo encontra-se na Tabela 1. Todas as disfunções foram qualificadas de discreta a moderada e sem sinais de repercussão clínica.

As variáveis ecocardiográficas quantitativas analisadas através da análise de variância, entre os cinco anos, não foram encontradas diferenças significativas. As flutuações dos seus resultados não permitiram a caracterização de grupos de pacientes que apresentassem tendência de aumento, redução ou manutenção destes parâmetros. A média dos resultados obtidos das variáveis estudadas encontram-se expressas na Tabela 2.

\section{2 - Avaliação do Estudo Hemodinâmico}

As médias das variáveis hemodinâmicas analisadas e da FEcat encontram-se descritas na Tabela 3. Em todas elas, comparando-se os resultados através da análise de variância, entre os cinco anos, não foram encontradas diferenças significativas. Da mesma forma que na avaliação ecocardiográfica, não foi possível o estabelecimento de grupos de pacientes que apresentassem tendência de aumento, redução ou manutenção dos parâmetros analisados. A única variável que apresentou diferenças significativas foi o IC. Aplicando-se, então, a análise de variância de dados repetidos, teste de comparações múltiplas (Newman-Keuls), entre os cinco anos (A1, A2, A3, A4 e A5), somente houve diferenças significativas nas comparaçōes entre:

$$
\begin{aligned}
& Q(A 1, A 3) \operatorname{com} F=4,500 \text { e } p=0,0324 ; \\
& Q(A 1, A 4) \operatorname{com} F=4,712 \text { e } p=0,0297 \\
& Q(A 1, A 5) \operatorname{com} F=5,259 \text { e } p=0,0215
\end{aligned}
$$

A distribuição percentual de coronariopatia nos pacientes em função do tempo de evolução pósoperatória acha-se representada no Gráfico 1. Notase que a incidência de lesão coronária aumentou progressivamente, quadruplicando do primeiro para o quinto ano de seguimento clínico. No quinto ano de controle (A5) $44,4 \%$ dos pacientes apresentavam algum grau de lesão obstrutiva evidente na angiografia coronária.

\section{3 - Avaliação da função do VE e a Rejeição}

As médias das variáveis, FEeco, FEcat e CP, analisadas no final de $A 3$, dos Grupos $A$ ( 5 rejeições) e B (5 rejeições), encontram-se discriminadas na Tabela 4. A análise comparativa das diversas variáveis não mostrou diferenças significativas entre os dois grupos.

\section{4 - Avaliação da Função do VE e a Etiologia da Miocardiopatia}

As médias das variáveis, FEeco, FEcat, e CP, analisadas no final de $\mathrm{A} 3$, dos Grupos $\mathrm{C}$ (Isquêmica) e D (Dilatada) encontram-se discriminadas na Tabela 5 . Não foram encontradas diferenças significativas nas diversas variáveis estudadas entre estes dois grupos de pacientes.

\begin{tabular}{|c|c|c|c|c|c|c|}
\hline & A1 & $A 2$ & $A 3$ & A4 & $A 5$ & $P$ \\
\hline VDF & $\begin{array}{r}107,7 \\
\pm 27,3\end{array}$ & $\begin{array}{r}117,4 \\
\pm 39,8\end{array}$ & $\begin{array}{r}119,6 \\
\pm 40,9\end{array}$ & $\begin{array}{r}126,9 \\
\pm 42,0\end{array}$ & $\begin{array}{r}108,9 \\
\pm 28,4\end{array}$ & 0,50 \\
\hline VSF & $\begin{array}{r}28,9 \\
\pm 11,8\end{array}$ & $\begin{array}{r}34,7 \\
\pm 18,9\end{array}$ & $\begin{array}{r}41,0 \\
+25,6\end{array}$ & $\begin{array}{r}41,7 \\
\pm 29,8\end{array}$ & $\begin{array}{r}32,9 \\
\pm 13,3\end{array}$ & 0,23 \\
\hline vs & $\begin{array}{r}79,0 \\
\pm 18,0\end{array}$ & $\begin{array}{r}82,8 \\
\pm 23,0\end{array}$ & $\begin{array}{r}78,7 \\
\pm 21,4\end{array}$ & $\begin{array}{r}85,2 \\
\pm 17,8\end{array}$ & $\begin{array}{r}76,1 \\
\pm 16,3\end{array}$ & 0,70 \\
\hline IMVE & $\begin{array}{r}119,3 \\
\pm 19,6\end{array}$ & $\begin{array}{r}121,5 \\
\pm 29,9\end{array}$ & $\begin{array}{r}130,2 \\
\pm 33,0\end{array}$ & $\begin{array}{r}138,8 \\
\pm 40,5\end{array}$ & $\begin{array}{r}136,3 \\
\pm 30,7\end{array}$ & 0,21 \\
\hline RVMVE & $\begin{array}{r}0,51 \\
\pm 0,12\end{array}$ & $\begin{array}{r}0,53 \\
\pm 0,14\end{array}$ & $\begin{array}{r}0,50 \\
\pm 0,14\end{array}$ & $\begin{array}{r}0,53 \\
\pm 0,18\end{array}$ & $\begin{array}{r}0,46 \\
\pm 0,09\end{array}$ & 0,56 \\
\hline FEeco & $\begin{array}{r}0,74 \\
\pm 0,06\end{array}$ & $\begin{array}{r}0,72 \\
\pm 0,07\end{array}$ & $\begin{array}{r}0,68 \\
\pm 0,11\end{array}$ & $\begin{array}{r}0,70 \\
\pm 0,11\end{array}$ & $\begin{array}{r}0,70 \\
\pm 0,06\end{array}$ & 0,71 \\
\hline
\end{tabular}

TABELA 2

MÉDIAS DAS VARIÁVEIS ECOCARDIOGRÁFICAS ESTUDADAS

VDF $(\mathrm{ml})=$ Volume distólico final; VSF $(\mathrm{ml})=$ Volume sistólico final; VS $(\mathrm{ml})=$ Volume sistólico; IMVE $\left(\mathrm{g} / \mathrm{m}^{2}\right)=$ Índice de massa de ventrículo esquerdo; RVMVE $(\mathrm{ml} / \mathrm{g})=$ Relação volume/massa do ventrículo esquerdo; $\mathrm{FEeco}=\mathrm{Fração} \mathrm{de} \mathrm{ejeção} \mathrm{determinada} \mathrm{pela}$ ecocardiografia. 
FIORELLI, A. I.; STOLF, N. A. G.; LEMOS, P. C. P.; POMERANTZEFF, P. M. A.; BOCCHI, E. A.; GRAZIOZI, P.; BUSNARDO, F.; GAIOTO, F.; GÓES, M. F. S.; JATENE, A. D. - Comportamento da função do ventrículo esquerdo a longo prazo no transplante cardiaco ortotópico. Rev. Bras. Cir. Cardiovasc., 8 (2):97-107, 1993.

TABELA 3

MÉDIAS DAS VARIÁVEIS HEMODINÂMICAS ESTUDADAS

\begin{tabular}{|c|c|c|c|c|c|c|}
\hline & $A 1$ & $A 2$ & $A 3$ & A4 & $A 5$ & $P$ \\
\hline PMA & $\begin{array}{r}130,6 \\
\pm 15,2\end{array}$ & $\begin{array}{r}130,2 \\
\pm 23,3\end{array}$ & $\begin{array}{r}124,2 \\
\pm 18,6\end{array}$ & $\begin{array}{r}125,9 \\
\pm 19,8\end{array}$ & $\begin{array}{l}118,0 \\
\pm 15,6\end{array}$ & 0,32 \\
\hline PCP & $\begin{array}{r}10,3 \\
\pm 3,6\end{array}$ & $\begin{array}{r}10,9 \\
\pm 3,5\end{array}$ & $\begin{array}{r}10,3 \\
\pm 3,1\end{array}$ & $\begin{array}{r}10,7 \\
\pm 3,8\end{array}$ & $\begin{array}{r}12,4 \\
\pm 4,5\end{array}$ & 0,05 \\
\hline pd2VE & $\begin{array}{r}12,9 \\
\pm 7,0\end{array}$ & $\begin{array}{r}12,8 \\
\pm 6,7\end{array}$ & $\begin{array}{r}11,3 \\
\pm 4,1\end{array}$ & $\begin{array}{r}14,2 \\
\pm 9,6\end{array}$ & $\begin{array}{r}13,9 \\
\pm 5,2\end{array}$ & 0,81 \\
\hline RVS & $\begin{array}{r}1877 \\
\pm 413\end{array}$ & $\begin{array}{r}2073 \\
\pm 501\end{array}$ & $\begin{array}{r}2112 \\
\pm 525\end{array}$ & $\begin{array}{r}2074 \\
\pm 399\end{array}$ & $\begin{array}{r}1884 \\
\pm 333\end{array}$ & 0,33 \\
\hline IC & $\begin{array}{r}3,00 \\
\pm 0,50\end{array}$ & $\begin{array}{r}2,74 \\
\pm 0,45\end{array}$ & $\begin{array}{r}2,58 \\
\pm 0,53\end{array}$ & $\begin{array}{r}2,60 \\
\pm 0,45\end{array}$ & $\begin{array}{r}2,58 \\
\pm 0,44\end{array}$ & 0,02 \\
\hline IS & $\begin{array}{r}34,3 \\
\pm 6,0\end{array}$ & $\begin{array}{r}32,2 \\
\pm 7,3\end{array}$ & $\begin{array}{r}30,2 \\
\pm 6,8\end{array}$ & $\begin{array}{r}31,3 \\
\pm 5,7\end{array}$ & $\begin{array}{r}30,8 \\
\pm 5,7\end{array}$ & 0,30 \\
\hline ITSVE & $\begin{array}{r}56,6 \\
\pm 11,9\end{array}$ & $\begin{array}{r}53,1 \\
\pm 21,2\end{array}$ & $\begin{array}{r}46,8 \\
\pm 12,7\end{array}$ & $\begin{array}{r}50,2 \\
\pm 11,4\end{array}$ & $\begin{array}{r}47,0 \\
\pm 12,3\end{array}$ & 0,19 \\
\hline FEcat & $\begin{array}{r}0,72 \\
\pm 0,11\end{array}$ & $\begin{array}{r}0,72 \\
\pm 0,09\end{array}$ & $\begin{array}{r}0,72 \\
\pm 0,09\end{array}$ & $\begin{array}{r}0,73 \\
\pm 0,16\end{array}$ & $\begin{array}{r}0,72 \\
\pm 0,18\end{array}$ & 0,99 \\
\hline
\end{tabular}

PMA $(\mathrm{mmHg})=$ Pressão média da aorta; $\mathrm{PCP}(\mathrm{mmHg})=$ Pressão de capilar pulmonar; $\mathrm{pd2VE}(\mathrm{mmHg})=$ Pressão diastólica final de ventrículo esquerdo; RVS (dinas. $\left.\mathrm{seg}, \mathrm{cm}^{-5}\right)=$ Resistência vascular sistêmica; IC $\left(\mathrm{ml} / \mathrm{m}^{2}\right)=$ Índice cardiaco; IS $\left(\mathrm{ml} / \mathrm{bat}, \mathrm{m}^{2}\right)=$ Índice sistólico; ITSVE $\left(\mathrm{g} \cdot \mathrm{m} \cdot \mathrm{m}^{2}\right)$ = Índice do trabalho sistólico do ventrículo esquerdo; FEcat = Fração de ejeção determinada pela ventriculografia.

GRÁFICO 1

INCIDÊNCIA DE CORONARIOPATIA

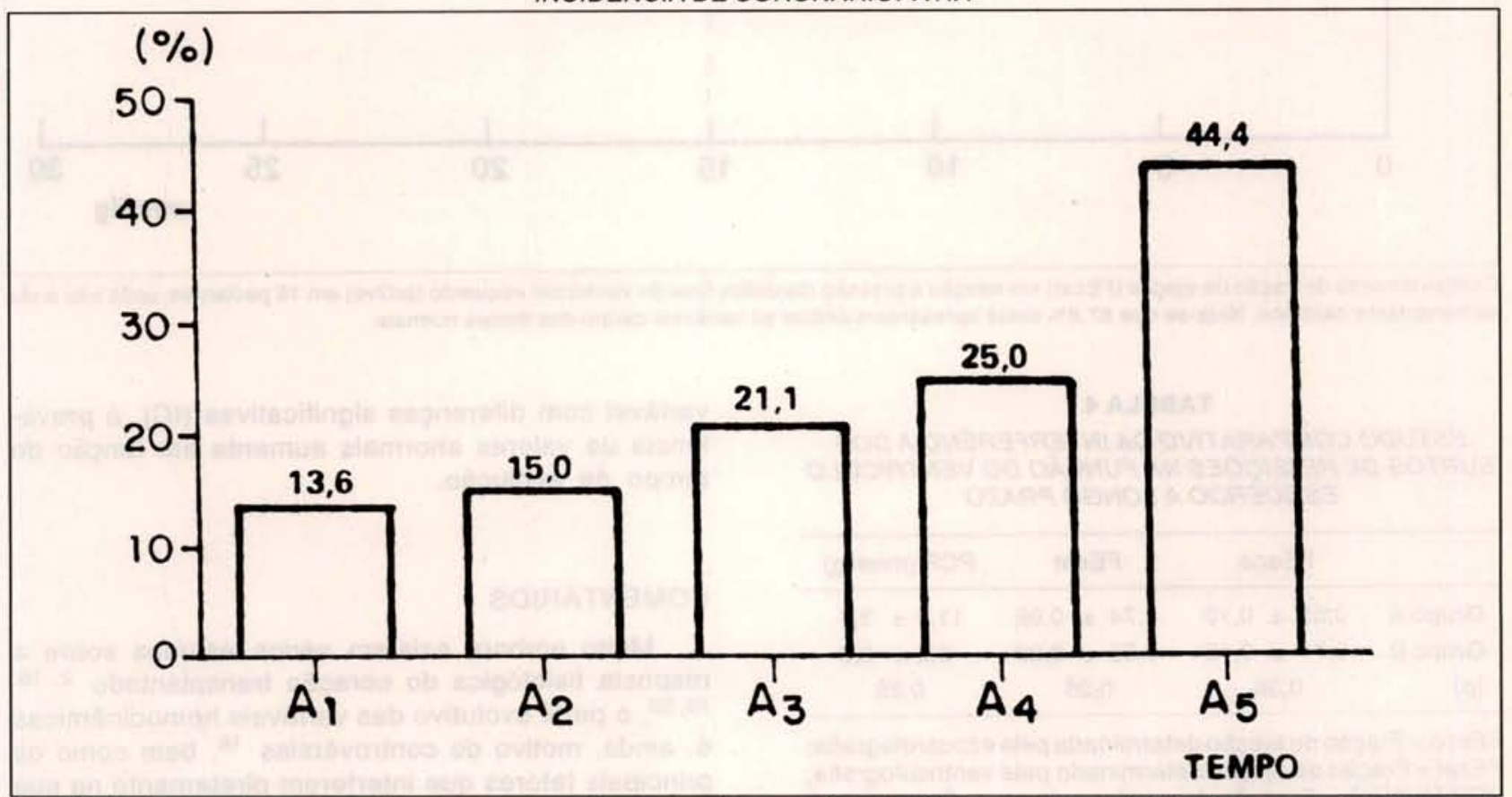

A incidência de aterosclerose coronária aumenta progressivamente após o transplante cardíaco; nota-se que a incidência de coronariopatia aumenta de $13,6 \%$ no primeiro ano para $44,4 \%$ após cinco anos.

No Gráfico 2 acha-se representada a relação entre a FEcat e a pd2VE de 16 (72,7\%) pacientes ao final de A3. Admitindo-se como anormais os valores da FEcat abaixo de 0,50 e da
pd2VE acima de $15 \mathrm{mmHg}$, pode-se notar que todos os pacientes apresentavam FEcat normais e apenas $2(12,5 \%)$ apresentavam pd2VE aumentada. 
FIORELLI, A. I.; STOLF, N. A. G.; LEMOS, P. C. P.; POMERANTZEFF, P. M. A.; BOCCHI, E. A.; GRAZIOZI, P.; BUSNARDO, F.; GAIOTO, F.; GÓES, M. F. S.; JATENE, A. D. - Comportamento da função do ventrículo esquerdo a longo prazo no transplante cardiaco ortotópico. Rev. Bras. Cir. Cardiovasc., 8 (2):97-107, 1993.

GRÁFICO 2

FRAÇĀO DE EJEÇÃO - FECat

PRESSÃO DIASTÓLICA FINAL DE VENTRICCULO ESQUERDO

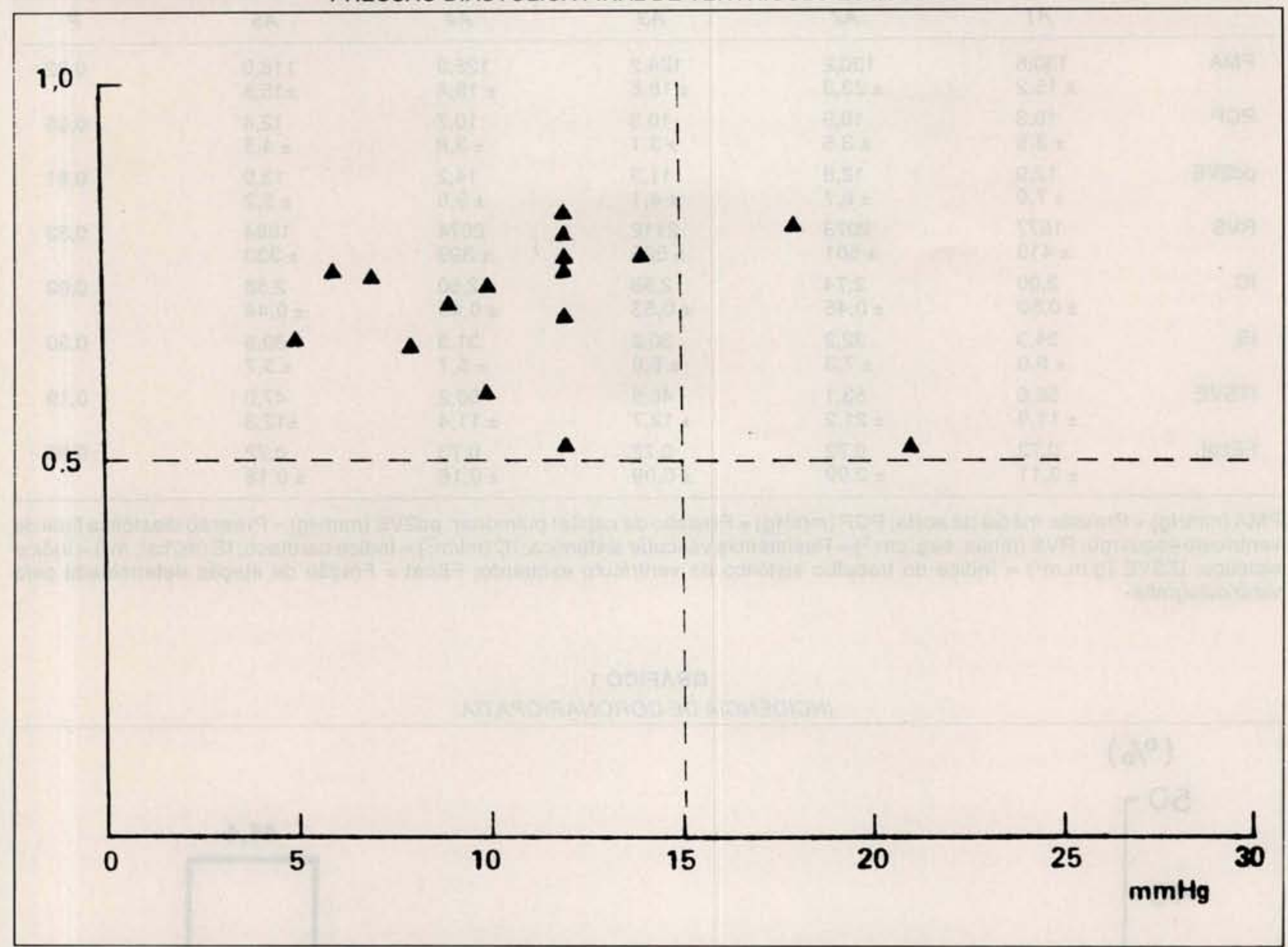

Comportamento da fração de ejeçăo (FEcat) em relação à pressão diastólica final do ventrículo esquerdo (pd2ve) em 16 pacientes, após três anos de transplante cardiaco. Nota-se que $87,8 \%$ deles apresentam ambas as variáveis dentro dos limites normais.

TABELA 4

ESTUDO COMPARATIVO DA INTERFERÊNCIA DOS SURTOS DE REJEIÇÕES NA FUNÇÄO DO VENTRICCULO ESQUERDO A LONGO PRAZO

\begin{tabular}{lccc}
\hline & FEeco & FEcat & PCP $(\mathrm{mmHg})$ \\
\hline Grupo A & $0,65 \pm 0,10$ & $0,74 \pm 0,08$ & $11,0 \pm 3,5$ \\
Grupo B & $0,71 \pm 0,10$ & $0,66 \pm 0,09$ & $9,2 \pm 2,3$ \\
(p) & 0,36 & 0,06 & 0,25 \\
\hline
\end{tabular}

FEeco = Fração de ejeção determinada pela ecocardiografia; FEcat $=$ Fração de ejeção determinada pela ventriculografia; PCP $(\mathrm{mmHg})=$ Pressão de capilar pulmonar; Grupo $A \geqslant 5$ rejeiçōes; Grupo B $<5$ rejeiçōes.

A distribuição da incidência de valores anormais das principais variáveis aferidas, durante o período da investigação acha-se apresentada na Tabela 6. Nota-se que, apesar de se ter encontrado uma única variável com diferenças significativas (IC), a prevalência de valores anormais aumenta em função do tempo de evolução.

\section{COMENTÁRIOS}

Muito embora existam vários estudos sobre a resposta fisiológica do coração transplantado 2,16 , 19,22 , o perfil evolutivo das variáveis hemodinâmicas é, ainda, motivo de controvérsias ${ }^{16}$, bem como os principais fatores que interferem diretamente na sua função contrátil 12, 19, 27 .

Vários autores têm descrito os principais aspectos hemodinâmicos do pós-operatório imediato 5,6 , 9,29 do transplante cardíaco, e um número bem menor tem apresentado a evolução da função ventricular com seguimento ecocardiográfico e hemodinâmico a longo prazo $1,10,11,16,26$. 
FIORELLI, A. I.; STOLF, N. A. G.; LEMOS, P. C. P.; POMERANTZEFF, P. M. A.; BOCCHI, E. A.; GRAZIOZI, P.; BUSNARDO, F.; GAIOTO, F.; GÓES, M. F. S.; JATENE, A. D. - Comportamento da função do ventrículo esquerdo a longo prazo no transplante cardíaco ortotópico. Rev. Bras. Cir. Cardiovasc., 8 (2):97-107, 1993.

TABELA 5

ESTUDO COMPARATIVO DA INTERFERÊNCIA DA ETIOLOGIA DA MIOCARDIOPATIA NA FUNÇÃO DO VENTRÍCULO ESQUERDO A LONGO PRAZO

\begin{tabular}{lccc}
\hline & FEeco & FEcat & PCP $(\mathrm{mmHg})$ \\
\hline $\begin{array}{l}\text { Grupo C } \\
\text { Isquêmica }\end{array}$ & $0,67 \pm 0,08$ & $0,69 \pm 0,08$ & $10,3 \pm 3,7$ \\
$\begin{array}{l}\text { Grupo D } \\
\text { Dilatada } \\
\text { (p) }\end{array}$ & $0,67 \pm 0,12$ & $0,72 \pm 0,13$ & $10,7 \pm 2,9$ \\
\hline
\end{tabular}

FEeco = Fração de ejeção determinada pela ecocardiografia; FEcat $=$ Fração de ejeção determinada pela ventriculografia; $\mathrm{PCP}(\mathrm{mmHg})=$ Pressão de capilar pulmonar.

A imunossupressão reveste-se de efeitos colaterais, ressaltando-se a nefrotoxicidade e a hipertensão arterial 2,17 , sendo que esta última tem como conseqüência a hipertrofia miocárdica, observada ao ecocardiograma e ao exame anatomopatológico. Por outro lado, a agressão imunológica crônica à íntima do vaso compromete as artérias coronárias, predispondo ao aparecimento da isquemia miocárdica silenciosa, que pode causar disfunções tardias no coração transplantado $3,10,15$.

\section{1 - Ecocardiografia}

Na nossa investigação, a análise qualitativa das valvas cardíacas mostrou que a insuficiência foi a disfunção valvar predominante, porém de pequena magnitude e sem sinais de repercussão clínica. As valvas mais acometidas foram a tricúspide e a mitral. Estas anormalidades têm sido documentadas de forma similar por outros autores, que apontam a distorção das cavidades atriais e o assincronismo dos batimentos atriais como os principais fatores responsáveis 1,20 .

O crescimento da cavidade ventricular, observado através da evolução do VDF e do VSF, fez parte das alterações que o coração transplantado sofreu durante o processo de adaptação, porém sem prejuízo do VS. Os sinais ecocardiográficos de hipertrofia do VE podem ser confirmados também através da análise do IMVE e da RVMVE. O IMVE confirmou a presença da hipertrofia na maioria dos exames ecocardiográficos realizados $(91 \%)$, ao contrário da RVMVE que identificou em apenas $63 \%$ dos exames. Esta distribuição mais homogênea na RVMVE sugere que, durante o processo de adaptaçäo do coração, houve um equilíbrio entre o aumento da cavidade do VE (VDF) e a hipertrofia (IMVE).

SASAYAMA et alii ${ }^{25}$, em 1976, confirmaram, em preparações isoladas, que a hipertrofia do VE pode ocorrer precocemente, porém os mecanismos intrínsecos que determinam essas alterações imediatas ao nível da massa do VE, do VDF e da FEeco são incertos 16 .

Apoiando-se na ecocardiografia, verifica-se que não houve mudanças na FEeco e na anatomia do coração transplantado do primeiro ao quinto ano de seguimento. Este fato está de acordo com os resultados obtidos por alguns autores ${ }^{16}$, que confirmam a estabilidade do coração transplantado, embora existam controvérsias a este respeito ${ }^{1}$.

\section{2 - Estudo Hemodinâmico}

Após um ano de transplante, todos os pacientes apresentavam a PSAP, a RVP, a PCP e a pd2VE dentro dos limites da normalidade e mantiveram-se sem alterações significativas até o quinto ano de controle. Apenas 1 paciente apresentou tendência de aumento da RVP, possivelmente como resposta à disfunção progressiva do coração transplantado.

A incidência de medidas anormais da PCP e da pd2VE aumentou com o passar do tempo. Provavelmente, este fato indique, de maneira indireta, uma lenta deterioração da função do VE a longo prazo. Elevações na PAP e na PCP têm sido observadas nos pacientes submetidos ao transplante durante 0 exercício ou com sobrecarga de volume ${ }^{16}$.

Dos $6(25 \%)$ pacientes que apresentaram pelo menos uma determinação da PCP acima de 15 $\mathrm{mmHg}$, em apenas $2(33,3 \%)$ identificaram-se lesões críticas nas artérias coronárias, através da angiografia e com comprometimento da FEcat. Verifica-se, portanto, que não existe uma correlação estreita entre a gravidade das lesões coronárias e a função do VE.

A hipertensão arterial tem sido uma constante no pós-operatório do transplante e apontada, de

TABELA 6

INCIDÊNCIA DE VALORES ANORMAIS NAS PRINCIPAIS VARIÁVEIS ESTUDADAS

\begin{tabular}{lrrrrr}
\hline & $\begin{array}{r}\text { A1 } \\
\%\end{array}$ & $\begin{array}{c}\text { A2 } \\
\%\end{array}$ & $\begin{array}{c}\text { A3 } \\
\%\end{array}$ & $\begin{array}{c}\text { A4 } \\
\%\end{array}$ & \multicolumn{1}{c}{$\begin{array}{c}\text { A5 } \\
\%\end{array}$} \\
\hline LC (*) & 13,6 & 15,0 & 21,1 & 25,0 & 44,4 \\
FEeco & 00,0 & 4,2 & 12,5 & 12,5 & 00,0 \\
FEcat & 10,5 & 11,1 & 17,6 & 20,0 & 25,0 \\
IC $\left(\mathrm{ml} / \mathrm{min}^{*} \mathrm{~m}^{2}\right)$ & 37,5 & 60,7 & 77,3 & 66,7 & 70,0 \\
PCP $(\mathrm{mmHg})$ & 8,3 & 4,1 & 9,1 & 13,3 & 25,0 \\
IMVE $\left(\mathrm{g} / \mathrm{m}^{2}\right)$ & 87,5 & 87,5 & 91,7 & 93,8 & 100,0 \\
PAM $\left(\mathrm{mmHg}^{2}\right)$ & 83,3 & 78,3 & 63,6 & 80,0 & 50,0 \\
RVS (dinas. seg, $\left.\mathrm{cm}^{-5}\right)$ & 91,7 & 91,3 & 95,5 & 93,3 & 90,9 \\
\hline
\end{tabular}

(*) - Lesão Coronária 
FIORELLI, A. I.; STOLF, N. A. G.; LEMOS, P. C. P.; POMERANTZEFF, P. M. A.; BOCCHI, E. A.; GRAZIOZI, P.; BUSNARDO, F.; GAIOTO, F.; GÓES, M. F. S.; JATENE, A. D. - Comportamento da função do ventrículo esquerdo a longo prazo no transplante cardiaco ortotópico. Rev. Bras. Cir. Cardiovasc., 8 (2):97-107, 1993.

maneira incerta, como fator adicional na deterioração da função do miocárdio $10,12,28$. GREENBERG et alii ${ }^{12}$, em 1985 , encontraram hipertensão arterial em $56 \%$ dos pacientes após um ano de transplante e aumento da pd2VE em $30 \%$. Na nossa casuística, pode-se observar a elevação da PAM e da RVS sem alterações significativas entre os diversos períodos estudados, a despeito do uso de hipotensores e diuréticos.

Os nossos resultados mostram que a função do VE, analisada através do IS e do ITSVE, não apresentou diferenças estatisticamente significativas até o quinto ano de evoluçäo. Porém, nota-se que os valores médios do IS estão ligeiramente deprimidos em relação aos níveis normais. No transplante, a FC em repouso normalmente está elevada, devido à ausência do tônus vagal ${ }^{22}$; possivelmente este fato participe no mecanismo de manutenção do IC em niveis adequados.

Ao contrário dos demais parâmetros analisados neste estudo, os resultados do IC mostraram depressão estatisticamente significativa dos valores médios obtidos no primeiro ano em relação ao quinto ano. A análise de variância de dados repetidos mostrou que estas diferenças, em relação à primeira determinação (A1), tornaram-se mais acentuadas a partir do terceiro ano de evolução (A3). A hipertensão arterial persistente e a incidência crescente de aterosclerose, muito provavelmente, desempenharam importante papel no aparecimento desta anormalidade. Deve-se ressaltar, ainda, que o achado de uma única variável (IC) com diferenças significativas, entre os diversos parâmetros estudados, pode estar dentro do intervalo de erro adotado $(5 \%)$.

Finalizando, sob o ponto de vista hemodinâmico, os nossos resultados mostram que, após cinco anos de transplante, o VE apresenta conservação da sua função. Porém, o aparecimento de redução significativa do IC associado à incidência crescente de anormalidades nas variáveis estudadas sugere que, para observações mais prolongadas, muito provavelmente as alterações tornem-se mais acentuadas.

\section{3 - Cinecoronariografia}

A aterosclerose acelerada e de caráter silencioso é uma complicação tardia no transplante cardíaco, devida à desnervação, e compromete a sobrevida a longo prazo dos pacientes 3,13 . Nas formas mais graves, a sua manifestação clínica usual é a disfunção do VE. Nos nossos pacientes a incidência da aterosclerose coronária no primeiro ano de evolução foi de $13,6 \%$, aumentando progressivamente até a $44,4 \%$ no quinto ano. Estes dados são comparáveis aos referidos na literatura $2,11,13$ e não parece terem-se modificado na sua incidência, após o uso da ciclosporina $2,11,13$.

A lesão coronária ocorreu em $6(27,3 \%)$ pacientes durante o período de estudo, com caráter progressivo, exceto em $1(16,7 \%)$ paciente que evoluiu com lesão severa desde o primeiro ano de pós-operatório. Neste subgrupo, $3(50 \%)$ pacientes apresentaram lesão coronária associada a importante redução da FEeco, FEcat e IC. Este fato mostra que a lesão aterosclerótica é um dos fatores que tardiamente pode comprometer a função do VE. Comportamento semelhante foi observado por VON SCHEIDT et alii ${ }^{28}$, em 1991, que mostraram a tendência de aumento da pd2VE nos portadores de coronariopatia.

\section{4 - Ventriculografia}

A FEcat manteve-se dentro dos limites da normalidade, sem diferenças significativas no período estudado. Contudo, a análise individual dos pacientes permitiu identificar, em $6(27,2 \%)$ deles, FEcat inferior a 0,50 .

Em 3 pacientes a FEcat encontrava-se deprimida desde o primeiro ano após o transplante. Notase que, em 2 pacientes, associou-se o desenvolvimento precoce de lesões graves nas coronárias, sendo, por este motivo, indicado transplante. Um paciente faleceu no $41^{\circ}$ mês de evolução, aguardando doador, e o outro não aceitou a nova operação. Apenas 1 paciente não apresentou alterações significativas na cinecoronariografia que justificassem a hipocinesia acentuada do VE. Tem-se atribuído estas anormalidades a possível seqüela do coração do doador, apesar do ecocardiograma e do cateterismo não terem detectado alterações.

Três pacientes apresentaram redução da FEcat no quinto ano de evolução, não se correlacionando com lesões significativas na cinecoronariografia. Possivelmente, algum comprometimento da microcirculação tenha sido responsável por esta redução da FEcat.

$\mathrm{Na}$ correlação entre a FEcat e a pd2VE no terceiro ano de evolução, verifica-se que todos os pacientes apresentavam FEcat dentro dos limites normais e que apenas em $2(12,5 \%)$ a pd2VE estava aumentada. Estes resultados são comparáveis a outros descritos na literatura caracterizando bom desempenho tardio do VE $6,10,22$; porém, tem-se encontrado redução da fração de ejeção para valores limites de 0,50 , mesmo após um ano de transplante 14

Desse modo, refletindo-se sobre exposto, podese inferir que a função do VE, observada através da 
FIORELLI, A. I.; STOLF, N. A. G.; LEMOS, P. C. P.; POMERANTZEFF, P. M. A.; BOCCHI, E. A.; GRAZIOZI, P.; BUSNARDO, F.; GAIOTO, F.; GÓES, M. F. S.; JATENE, A. D. - Comportamento da funçāo do ventrículo esquerdo a longo prazo no transplante cardíaco ortotópico. Rev. Bras. Cir. Cardiovasc., 8 (2):97-107, 1993.

FEcat, tende a se manter estável a longo prazo, sendo a doença aterosclerótica um importante fator que interfere negativamente no seu desempenho. Convém destacar também que, no coração transplantado, a agressão coronária geralmente é difusa, iniciando-se nos vasos de pequeno calibre, com prejuízo da microcirculação. Este fato poderia explicar a deterioração lenta da FEcat, precedendo muitas vezes as lesões em artérias de maior calibre visíveis na angiografia, assim como a morte súbita, observada em um dos nossos pacientes.

\section{5 - Função do VEX Rejeição}

A ciclosporina- $A$, reduzindo o edema do miocárdio, modificou o comportamento hemodinâmico nos episódios de rejeição. As alterações nas variáveis hemodinâmicas, durante estes episódios, antes eram mais pronunciadas 5,19 , confirmando que, atualmente, estes parâmetros tornaram-se índices pouco expressivos no diagnóstico da rejeição ${ }^{16}$.

Os nossos dados mostram que o número de rejeições agudas (moderada ou severa) não interferiram significativamente na função tardia do VE; estes resultados não diferem de outros apresentados na literatura 10,16 .

\section{6 - Função do VE x Etiologia da Miocardiopatia}

No presente estudo não houve diferenças significativas entre as variáveis escolhidas para avaliar a função do VE e a origem das miocardiopatias, comparáveis aos de outros autores $11,13,18$.

LANZA et alii ${ }^{18}$, em 1984, não encontraram interferência significativa da etiologia da miocar- diopatia na evolução tardia dos pacientes submetidos ao transplante, sugerindo apenas que, na doença reumática, a sobrevida é mais favorável;. Por outro lado, em relatos anteriores, temos referido que os pacientes com doença de Chagas têm apresentado maior morbidade e mortalidade em relação aos demais 8 .

\section{7 - Função do VE x Coronariopatia}

No estudo comparativo da função do VE, com a presença ou não de coronariopatia, não foi possível a aplicação de um tratamento estatístico adequado, tendo em vista o reduzido número de pacientes. Porém, os resultados sugerem que a doença aterosclerótica tende a deteriorar, progressivamente, a função do VE após o transplante. Torna-se necessário aumentar o número de pacientes estudados e o tempo de investigação para confirmação.

Concluindo, a análise a longo prazo das alterações anatômicas e funcionais que ocorrem no coração transplantado, estudadas evolutivamente com diferentes variáveis ecocardiográficas e hemodinâmicas, não mostrou diferenças estatisticamente significativas no período considerado, demonstrando que: 1) há conservação da função do VE até o quinto ano de evolução; 2) há hipertrofia do VE na adaptação ao novo sistema vascular; 3 ) os surtos agudos de rejeição e a etiologia da miocardiopatia não interferiram na função do VE tardiamente e 4) a aterosclerose acelerada exerce importante efeito deletério na função miocárdica. Contudo, a redução significativa do índice cardíaco e o aparecimento crescente de resultados anormais na FEeco e na FEcat, possivelmente indiquem uma tendência a um lento prejuízo da função tardia do VE. 
FIORELLI, A. I.; STOLF, N. A. G.; LEMOS, P. C. P.; POMERANTZEFF, P. M. A.; BOCCHI, E. A.; GRAZIOZI, P.; BUSNARDO, F.; GAIOTO, F.; GÓES, M. F. S.; JATENE, A. D. - Comportamento da função do ventrículo esquerdo a longo prazo no transplante cardíaco ortotópico. Rev. Bras. Cir. Cardiovasc., 8 (2):97-107, 1993.

\section{RBCCV 44205-204}

FIORELLI, A. I.; STOLF, N. A. G.; LEMOS, P. C. P.; POMERANTZEFF, P. M. A.; BOCCHI, E. A.; GRAZIOZI, P.; BUSNARDO, F.; GAIOTTO, F.; GÓES, M. F. S.; JATENE, A. D. - Long-term behaviour of left ventricular function in ortothopic heart transplantation. Rev. Bras. Cir. Cardiovasc., 8 (2): 97-107, 1993.

ABSTRACT:Ortothopic heart transplantation (OHT) was performed in 110 patients at the Heart Institute, between march 1985 and february 1993 for treatment of terminal cardiomyopathy. From these patients, 24 had a survival period of more than 36 months and were selected for a long term analysis of the left ventricular function. They were observed in a 56 months average follow-up. The patients were 44.3 years old average, being $21(87.5 \%)$ male. The ethiology of the cardiac diseases was dilated cardiomyopathy for $13(54.2 \%)$ patients, ischemic for $8(33.3 \%)$ patients and Chagas' Disease for $3(12.5 \%)$ patients. All patients were initially in functional class IV (NYHA). They were evaluated by cinecoronariography, hemodynamic and echocardiographic studies preoperatively, and every year in a 5 years follow-up. The results suggest that: 1) all patients presented left ventricular hipertrophy as an adaptative mechanism;2) the arterial hypertension was observed since the first year of follow-up; 3 ) the acute rejection episodes and the myocardiopathy ethiology did not interfere on the left ventricular function; 4) the acelerated atherosclerosis had a negative effect on left ventricular function; 5) the echocardiographic and hemodynamic evaluation showed a left ventricular function stability of 5 years after surgery; nevertheless, it was observed an important reduction of the cardiac index associated with a crescent increase of abnormal measures results, suggesting a late reduction of the left ventricular performance.

DESCRIPTORS: heart transplantation, ortothopic; heart transplantation, left ventricular function.

\section{REFERÊNCIAS BIBLIOGRÁFICAS}

1 ANTUNES, M. L.; SPOTNITZ, H. M.; CLARK, M. B.; STEINHARDT, M. J.; MARBOE, C. C.; SMITH, C. R.; ROSE, E. A.; REEMTSMA, K. - Long-term function of human cardiac allografts assessed by twodimensional echocardiography. J. Thorac. Cardiovasc. Surg., 98: 275-284, 1989.

2 BIEBER, C. P.; HUNT, S.; SCHWINN, D. A.; JAMIESON, S. W.; REITZ, B. A.; OYER, P. E.; SHUMWAY, N. E.; STINSON, E. B. - Complications in long-term survivors of cardiac transplantation. Transplant. Proc., 13: 207-211, 1981.

3 BILLINGHAM, M. E. - Cardiac transplant atherosclerosis. Transplant. Proc., 19: 19-25, 1987.

CARREL, A. \& GUTHRIE, C. C. - The transplantation of veins and organs. Am. Med., 10: 1101-1102, 1905.

5 CHARTRAND, C.; DONG Jr., E.; SHUMWAY, N. E. Hemodinamics of the orthotopic homotransplanted heart. Surg. Forum, 19: 238-239, 1968.

6 CORCOS, T.; TAMBURINO, C.; LEGER, P.; VASSIER, E.; ROSSANT, P.; MATTEI, M. F.; DAUDON, P.; GANDJBAKHCH, I.; PAVIE, A.; CABROL, A.; CABROL, C. - Early and late hemodynamic evaluation after cardiac transplantation: a study of 28 cases. $J$. Am. Coll. Cardiol., 11: 264-269, 1988.

DE BACKEY, M. E.; DIETHRICH, E. B.; GLICK, G.; NOON, G. P.; BUTLER, W. T.; ROSSEN, R. D.; LIDDICOAT, J. E.; BROOKS, D. K. - Human cardiac transplantation: clinical experience. Cardiovasc. Surg., 58: 303-317, 1969.
8

10
FIORELLI, A. I.; STOLF, N. A. G.; BOCCHI, E. A.; SEFERIAN, P.; HIGUSHI, L.; UIP, D.; STRABELLI, T.; KALIL, J.; NEWMANN, J.; JATENE, F. B. POMERANTZEFF, P. M. A.; LEMOS, P. C. P.; PEREIRA-BARRETO, A. C.; BELLOTTI, G.; JATENE, A. D. - Evoluçăo tardia do transplante cardiaco na doença de Chagas. Rev. Bras. Cir. Cardiovasc., 5: 113-119, 1990.

9 FIORELLI, A. I.; STOLF, N. A. G.; MORAES, A. V.; PASCUAL, J.; AULER Jr., J. O.; LEMOS, P. C.; POMERANTZEFF, P.; JATENE, F.; BOCCHI, E.; BARRETO, A. C. P.; BELLOTTI, G.; JATENE, A. D. - Avaliação da contratilidade direita no transplante cardiaco. In: Congresso Nacional de Cirurgia Cardiaca, 16., Săo Paulo, 1989. (Resumos dos trabalhos). Săo Paulo, 1989. p. 36.

0 FRIST, W. H.; STINSON, E. B.; OYER, P. E.; BALDWIN, J. C.; SHUMWAY, N. E. - Long-term hemodynamic results after cardiac transplantation. J. Thorac. Cardiovasc. Surg., 94: 685-693, 1987.

1 GRATTAN, M. T.; MORENO-CABRAL, C. E.; STARNES, V. A.; OYER, P. E.; STINSON, E. B.; SHUMWAY, N. E. - Eight-year results of cyclosporine treated patients with cardiac transplants. J. Thorac. Cardiovasc. Surg., 99: 500-509, 1990.

GREENBERG, M. L.; URETSKY, B. F.; REDDY, P. S.; BERNSTEIN, R. L.; GRIFFITH, B. P.; HARDESTY, R. L.; THOMPSON, M. E.; BAHNSON, H. T. - Long- 
FIORELLI, A. I.; STOLF, N. A. G.; LEMOS, P. C. P.; POMERANTZEFF, P. M. A.; BOCCHI, E. A.; GRAZIOZI, P.; BUSNARDO, F.; GAIOTO, F.; GÓES, M. F. S.; JATENE, A. D. - Comportamento da função do ventrículo esquerdo a longo prazo no transplante cardíaco ortotópico. Rev. Bras. Cir. Cardiovasc., 8 (2):97-107, 1993.

term hemodynamic follow-up of cardiac transplant patients treated with cyclosporine and prednisone. Circulation, 71: 487-494, 1985. B. A.; COPELAND, J. G.; OYER, P. E.; SHUMWAY, N. E. - Control of graft arteriosclerosis in human heart transplant recipients. Surgery, 81: 262-269, 1977.

GROSSMAN, W; BRAUNWALD, E.; MANN, T.; McLAURIN, L. P.; GREEN, L. H. - Contractile state of the left ventricle in man as evaluated from end systolic pressure volume relations. Circulation, 56: 845-846, 1977.

HALLMAN, G. L.; LEATHERMAN, L. L.; LEACHMAN, R. D.; COOLEY, D. A. - Performance of the transplanted heart. J. Cardiovasc. Surg., 11: 1-6, 1970.

HOSENPUD, J. D.; MORTON, M. J.; WILSON, R. A.; PANTELY, G. A.; NORMAN, D. J.; COBANOGLU, M. A.; STARR, A. - Abnormal exercise hemodynamics in cardiac allograft recipients 1 years after cardiac transplantation. Circulation, 80: 525-532, 1989.

17 KAHAN, B. D. - Cyclosporine nephrotoxicity: pathogenesis, prophylaxis, therapy, and prognosis. Am. J. Kidney Dis., 8: 323-331, 1986.

18 LANZA, R. P.; COOPER, D. K.; BOYD, S. T.; BARNARD, C. N. - Comparison of patients with ischemic, myopathic, and rheumatic heart diseases as cardiac transplantat recipients. Am. Heart J., 107: 8-12, 1984.

19 LEACHMAN, R. D.; COKKINOS, D. V.; ROCHELLE, D. G.; ZAMALLOA, O.; MILAM, J. D.; HALLMAN, G. L.; COOLEY, D. A. - Serial hemodynamic study of the transplanted heart and correlation with clinical rejection. J. Thorac. Cardiovasc. Surg., 61: 561$569,1971$.

20 LEWEN, M. K.; BRYG, R. J.; MILLER, L. W.; WILLIAMS, G. A.; LABOVITZ, A. J. - Tricuspid regurgitation by Doppler echocardiography after orthotopic cardiac transplant. Am. J. Cardiol., 59: 1371-1374, 1987.

OLIVARI, M. T.; ANTOLICK, A.; RING, W. S. - Arterial

hypertension in heart transplant recipients treated with triple-drug immunosupressive therapy. J. Heart Transplant., 8: 34-39, 1989.

PFLUGFELDER, P. W.; McKENZIE, F. N.; KOSTUK, W. J. - Hemodynamics profiles at rest and during supine exercise after orthotopic cardiac transplantation. Am. J. Cardiol., 61: 1328-1333, 1988.

SAHN, D. J.; DeMARIA, A.; KISSLO, J.; WEYMAN, A. - Recomendations regarding quantitation in M-Mode echocardiography: results of a survey of echocardiographic measurements. Circulation, 58: 1072-1083, 1978.

SAS, Institute Inc.; SAS/STAT Guide Personal Computers, Version 6 Edition Cary, NC: SAS Institute Inc. 1987.

SASAYAMA, S.; ROSS Jr., J.; FRANKLIN, D.; BLOOR, C. M.; BISHOP, S.; DILLEY, R. B. - Adaptations of the left ventricle to chronic pressure overload. Circ. Res., 38: 172-178, 1976.

STOLF, N. A. G.; FIORELLI, A. I.; BOCCHI, E. A.; PASCUAL, J. M.; AULER JÚNIOR, J. O. C.; LEMOS, P. C. P.; JATENE, F. B.; POMERANTZEFF, P. M.; BELLOTTI, G.; PILEGGI, F.; JATENE, A. D. - Evolução hemodinâmica seqũencial no transplante cardiaco. Rev. Bras. Circ. Cardiovasc., 3: 189$195,1988$.

TAMBURINO, C.; CORCOS, T.; FERACO, E.; LEGER, P.; DESRUENNES, M.; VAISSIER, E.; GANDJBAKHCH, I.; PAVIE, A.; CABROL, C. Hemodynamic parameters one and four weeks after cardiac transplantation. Am. J. Cardiol., 63: 635639, 1989.

VON SCHEIDT, W.; ZIEGLER, U.; KEMKES, B. M.; ERDMANN, E. - Heart transplantation: hemodynamics over a five year period. J. Heart Lung Transplant., 10: 342-350, 1991.

29 WAHLERS, T. H.; BEER, C.; FIEGUTH, H. G. JURMANN, N.; SCHAFERS, H. J.; HERRMAN, G.; FRIMPONG, K.; HAVERICH, A.; BORST, H. G. Right heart failure following orthotopic cardiac transplantation. Clin. Transplant., 2: 252-258, 1988. 